\title{
Effect of Nourishing "Yin"-Removing "Fire" Chinese Herbal Mixture on Hypothalamic NKB/NK3R Expression in Female Precocious Rats
}

\author{
Shiran Wang, ${ }^{1}$ Liting Zhu, ${ }^{1}$ Jian $\mathrm{Yu}^{2}{ }^{2}$ and Zhanzhuang Tian ${ }^{1}$ \\ ${ }^{1}$ Department of Neurobiology and Integrative Medicine, Shanghai Medical College, Fudan University, P.O. Box 291, \\ 138 Yi-Xue-Yuan Road, Shanghai 200032, China \\ ${ }^{2}$ Department of Integrative Medicine, Children's Hospital, Fudan University, 399 Wan Yuan Road, Shanghai 200032, China
}

Correspondence should be addressed to Zhanzhuang Tian; tianvv@shmu.edu.cn

Received 22 February 2014; Revised 21 May 2014; Accepted 25 May 2014; Published 16 June 2014

Academic Editor: Ping Li

Copyright (C) 2014 Shiran Wang et al. This is an open access article distributed under the Creative Commons Attribution License, which permits unrestricted use, distribution, and reproduction in any medium, provided the original work is properly cited.

Aim. The present study aims to investigate the effects of nourishing "Yin"-removing "Fire" Chinese herb mixture on the hypothalamic NKB/NK3R expression in female precocious model rats. Materials and Methods. Female Sprague-Dawley rats were randomly divided into four groups: normal $(\mathrm{N})$, central precocious puberty $(\mathrm{CPP})$ model $(\mathrm{M})$, CPP fed with Chinese herbal mixture $(\mathrm{CHM})$, and CPP fed with normal saline (MS). Rats on postnatal day 5 were given a single subcutaneous injection of $300 \mu \mathrm{g}$ to establish CPP model rats. Rats of CHM and MS groups were continuously administered with nourishing "Yin"-removing "Fire" Chinese herb mixture or saline since postnatal day 15. The expressions of hypothalamic NKB/NK3R were detected by means of real-time PCR, western blot, and immunofluorescence histochemistry. Results. The day of vaginal opening and establishment of two regular estrous cycles were delayed in the CHM group compared with M and MS groups. The expression of hypothalamic $\mathrm{NKB} / \mathrm{NK} 3 \mathrm{R}$ mRNA and protein in the arcuate nucleus (ARC) and medial preoptic (MPO) area were decreased significantly in the CHM group compared with the M and MS groups on the day of onset-puberty. Conclusions. These results indicate that the $\mathrm{NKB} / \mathrm{NK} 3 \mathrm{R}$ signaling pathway might be involved in the effect of herbal mixture treatment on CPP.

\section{Introduction}

Precocious puberty is defined by the development of sexual characters before the age of 8 years in girls and 9 years in boys. Idiopathic central precocious puberty $(\mathrm{CPP})$ is the most common type of sexual precocity [1]. It is usually due to the earlier activation of the hypothalamic GnRH neurons. Recently, a lot of studies found that NKB/NK3R signaling pathway plays a critical role in reproduction.

Neurokinin B (NKB) is a 10 amino acid peptide which belongs to the tachykinin family. Three tachykinin receptors are known: NK1, NK2, and NK3. NK3 receptor (NK3R) predominantly mediated the effects of NKB [2]. NKB and NK3R are widely spread throughout the central nervous system. Substantial studies supported that NKB/NK3R regulated $\mathrm{GnRH}$ neurons activation in hypothalamus during pubertal period [3]. The expression of Tac2 and Tacr3 mRNA (encoding NKB and NK3R, resp.) was increased along postnatal maturation in female rat hypothalamus [2]. Several studies found that, similar to GPR54 mutations, human mutations in the TAC and TACR3 (the genes encoding NKB and NK3R, resp.) might result in hypogonadotropic hypogonadism $(\mathrm{IHH})[4,5]$. Central administration of NKB agonist (senktide) to rats might induce a profound increase in serum levels of $\mathrm{LH}$, and this effect was regulated by E2-dependent negative feedback $[6,7]$. A dramatic increase in LH concentrations was observed after injection of senktide into the third ventricle during the follicular phase in ewes [8]. Single i.v. injection of NKB or senktide elicited robust LH discharges while they were abolished by GnRH receptor antagonism [9]. Recently, neurokinin B (NKB) and dynorphin (Dyn) were found to coexist within kisspeptin neurons in the arcuate 
nucleus (ARC) where they control the pulsatile release of GnRH $[10,11]$. These studies suggest that NKB might be another critical regulator of GnRH release and might involve in the advanced puberty onset in CPP.

$\mathrm{GnRH}$ analogue ( $\mathrm{GnRHa}$ ) has been recognized as an effective treatment for advanced puberty onset in CPP patients $[12,13]$. Treatment with GnRHa might inhibit the HPG axis of CPP patients, affecting the final height of children, and it would increase the probability of suffering from hyperandrogenism and PCOS when they grow up [13-15]. Traditional Chinese medicines have been successfully used for the management of CPP and improved the clinical symptoms for more than thirty years. The Chinese herb-based formulation nourishing "Yin"-removing "Fire" herbal mixture could downregulate the GnRH expression and significantly delay the sexual development of the precocious puberty rat [16]. It is reported that, Chinese herbal mixture could remarkably reduce the activity of $\mathrm{GnRH}$ neurons in the hypothalamus through inhibiting central excitatory amino acid neurotransmitter and promoting central inhibitory amino acid neurotransmitter and beta-endorphin release [17]. We have investigated that the herbal mixture decreased the kiss-1/GPR54 expression in female precocious rats [18]. However, the mechanism of NKB/NK3R signaling participation in the Chinese herb mixture therapeutic effect on precocious puberty is unclear. The present study aims to investigate the effects of nourishing "Yin"-removing "Fire" herbal mixture on hypothalamic NKB/NK3R expression in female precocious rats and further explores the therapeutic mechanism of the herbal mixture in CPP.

\section{Materials and Methods}

2.1. Animals and Drugs. Female Sprague-Dawley rats at postnatal day 3 (P3) with their mothers were purchased from Medical Experimental Animals Center of Chinese Academy of Sciences (Shanghai, China). Rats were housed under a $12: 12 \mathrm{~h}$ light/dark cycle with food and water available ad libitum. Animals were randomly divided into normal $(\mathrm{N})$, CPP model (M), CPP model administered with herb mixture (CHM), and CPP model administered with saline (MS) groups. On P5, all CPP model litters were given a single subcutaneous injection of $300 \mu \mathrm{g}$ of danazol (Hualian Pharm Ltd., Shanghai, China) dissolved in $25 \mu \mathrm{l}$ vehicle of glycol-ethanol $(1: 1, \mathrm{v} / \mathrm{v})$ [19]. From P15, rats in the CHM and MS were continuously gavaged with nourishing "Yin"removing "Fire" Chinese herbal mixture or saline $1 \mathrm{~mL} / 50 \mathrm{~g}$ body weight, until sacrificed. From P20, rats were inspected daily for vaginal opening (VO); thereafter, vaginal smears were examined daily to identify the period of estrous cycle, until two consecutive regular estrous cycles were established. After two consecutive regular estrous cycles, all animals were sacrificed by decapitation on diestrum estrous cycle with blood and hypothalamus tissue collected. The uterus and ovaries were dissected out and weighed to evaluate the organ coefficients $(\mathrm{mg} / 100 \mathrm{~g})$. The animals used in this study were in accordance with the NIH Guidelines and were approved by Animal Use and Care Committee for Fudan University.
The nourishing "Yin"-removing "Fire" Chinese herbal mixture prescription is mainly composed of 10 medicinal plants: $15 \mathrm{~g}$ of Rehmannia glutinosa (Sheng di), $9 \mathrm{~g}$ each of Scrophularia buergeriana (Xuan shen), Anemarrhena asphodeloides (Zhi mu), Cortex Phellodendri (Huang bai), Paeonia suffruticosa Andr. (Dan pi), Alisma plantago-aquatica L. var. orientale Sam. (Ze xie), Prunella vulgaris L. (Xia ku cao), $12 \mathrm{~g}$ of Carapax et Plastrum Testudinis (Gui jia), $30 \mathrm{~g}$ of Fructus hordei germinate (Mai ya), and $6 \mathrm{~g}$ of Gentiana scabra Bge (Long Dan Cao). All the above crude drugs were boiling gently in $1000 \mathrm{~mL}$ water for $40 \mathrm{~min}$ [18]. The mixture was kindly provided by the Department of Integrative Medicine, Children's Hospital of Fudan University, $60 \mathrm{~mL} /$ bottle $(1 \mathrm{~mL}$ containing $3 \mathrm{~g}$ of natural medicament power).

2.2. Hormone Assay by RIA. The blood samples of all the rats were collected from tail veins, respectively, at the time of sacrifice. The plasma was separated by centrifugation and stored at $-80^{\circ} \mathrm{C}$ until assayed. Concentration of $\mathrm{E}_{2}, \mathrm{LH}$, and FSH was determined by double-antibody RIA kits purchased from the Beijing Sinouk Institute of Biological Technology (Beijing, China). The samples were assayed in duplicate, and all the subjects' samples were assayed together. The sensitivity of the kit for E2 was less than $5 \mathrm{pg} / \mathrm{mL}$; the intra- and interassay coefficients were less than $10 \%$ and $15.2 \%$, respectively. For the sensitivity of the kit for LH, the assay sensitivity was $0.2 \mathrm{mIU} / \mathrm{mL}$, and the intra- and interassay coefficients of variation were $2.0-2.4 \%$ and $4.2-7.5 \%$, respectively. For the sensitivity of the kit for FSH, the assay sensitivity was $0.25 \mathrm{mIU} / \mathrm{mL}$, and the intra- and interassay coefficients of variation were $2.2-2.5 \%$ and $3.7-8.7 \%$, respectively.

2.3. Immunofluorescence Analysis. The effects of nourishing "Yin"-removing "Fire" Chinese herb mixture on the hypothalamic NKB and NK3R expression in different development stages of the rats were investigated. Hypothalamic samples from the four groups were obtained on the day of prepuberty (postnatal 21 days, $n=5$ per group), onset-puberty (the day of vaginal opening, about postnatal 24 days, $n=5$ per group), and postpuberty period (establishment of two regular estrous cycles, about postnatal 34 days, $n=5$ per group). The animals were exsanguinated with normal saline and followed with $4 \%$ paraformaldehyde in $0.1 \mathrm{M}$ phosphate buffer ( $\mathrm{PH} 7.4$ ). After perfusion was done, brains were removed and postfixed in $4 \%$ paraformaldehyde in $0.1 \mathrm{M}$ PBS (PH 7.4) with $30 \%$ sucrose, and their section was sliced at $30 \mu \mathrm{m}$ thickness on a vibratome microslicer stored at $4^{\circ} \mathrm{C}$ in tissue culture wells containing $0.1 \mathrm{M}$ PBS (PH 7.4) plus $0.02 \%$ sodium azide until further processed.

Slices were treated with $1 \%$ Triton X-100 firstly, washed in PBS for $30 \mathrm{~min}$ at room temperature (RT), and blocked with $10 \%$ normal donkey serum. Then, slices were incubated with a rabbit polyclonal antineurokinin $\mathrm{B}(\mathrm{NKB}, 1: 500$; Novus Biologicals, LLC, USA) or a rabbit polyclonal antineurokinin $B$ receptor (NK3R, 1:800; Novus Biologicals, LLC, USA) primary antibodies at $4^{\circ} \mathrm{C}$ for $24 \mathrm{~h}$. After rinsing, sections were incubated for $1 \mathrm{~h}$ at room temperature in the secondary antibody cocktail. For fluorescence detection of the NKB/NK3R 
antibodies, a 1:500 dilution of donkey anti-rabbit Alexa Fluor 488 was used. After secondary labeling, all slices were immediately rinsed and mounted onto subbed slides and coverslipped for analysis by fluorescence microscopy.

2.4. Real-Time Reverse Transcriptase-PCR (RT-PCR). For $\mathrm{NKB} / \mathrm{NK} 3 \mathrm{RmRNA}$ analysis, the target regions, including mediobasal hypothalamus and the suprachiasmatic-preoptic areas, were dissected on the onset-puberty period. The rat brains of all groups ( $n=5$ per group) were rapidly removed and hypothalamus was separated immediately and frozen in liquid nitrogen. Total hypothalamic RNA was extracted using “TRIzol Reagent" (Invitrogen Inc., America) according to the manufacturer's instructions. The purity and integrity of the RNA were checked spectroscopically and by gel electrophoresis before carrying out the analytical procedures.

The total RNA was digested with RNase-free DNase I (Invitrogen, Carlsbad, CA) before conducting real-time reverse transcriptase-PCR. To obtain cDNA, $2.0 \mu \mathrm{g}$ of total RNA was reverse transcribed using the SuperScript III reverse transcription system (Invitrogen Corp., Carlsbad, CA, USA) according to the manufacturer's prescriptions.

The primers used for NKB and NK3R mRNA analysis were designed and synthesized by Invitrogen with HPLC purity. Quantitative real-time PCR was carried out by IQ5 real-time PCR detection system (Bio-Rad, Richmond, CA). PCR assay linearity ranges were previously established for each gene cDNA to determine the sensitivity and efficiency of the amplification. The reactions were set up with $11.25 \mu \mathrm{l}$ SYBR Green RealMasterMix, $1.0 \mu$ l primer mixture (200 nM) and $0.5 \mu \mathrm{l}$ cDNA template. The thermal cycling conditions were as follows: $95^{\circ} \mathrm{C}$ for $2 \mathrm{~min}$ for denaturation, followed by 45 cycles of $95^{\circ} \mathrm{C}$ for $10 \mathrm{~s}$ and $60^{\circ} \mathrm{C}$ for $20 \mathrm{~s}$, and $72^{\circ} \mathrm{C}$ for $20 \mathrm{~s}$. After the cycles, a melting curve analysis was performed to insure purity of PCR products.

All real-time experiments were run in triplicate and a mean value was used for the determination of mRNA levels. Relative mRNA expression levels for NKB and NK3R were analyzed using the formula $2^{-\Delta \Delta \mathrm{Ct}}$ method and normalized to the $\beta$-actin ribosomal RNA.

2.5. Western Blot Analyses. On the day of onset-puberty in $M$, all rats of four groups were sacrificed by decapitation. NK3R protein expression was investigated by western blot with a standard procedure. After rapid removal of the brain, the hypothalamus has been removed, snapped frozen in liquid nitrogen, and stored at $-80^{\circ} \mathrm{C}$. For total protein extraction, hypothalamus was homogenized in $300 \mu \mathrm{l}$ RIPA lysis Buffer (Beyotime, China) with protease inhibitors (phenylmethanesulfonyl fluoride, Beyotime, China) using a Polytron homogenizer. Tissue lysates were cleared by centrifugation at 13,000 rpm for $15 \mathrm{~min}$ at $4^{\circ} \mathrm{C}$. Protein content was determined by the BCA method (Bio-Rad). Samples were boiled with loading buffer (1650 mM Tris- $\mathrm{HCl}, 2 \%$ SDS, $10 \%$ glycerol, $10 \% \beta$-mercaptoethanol, and $0.001 \%$ bromophenol blue) for $10 \mathrm{~min}$ and stored at $-80^{\circ} \mathrm{C}$ until use.

Protein aliquots were separated on $10 \%$ SDS polyacrylamide gels for $70 \mathrm{~min}$ at $110 \mathrm{~V}$. Then proteins were transferred onto polyvinyldifluoride membranes (Millipore, USA) in a Trans-Blot apparatus (Bio-Rad Laboratories, Inc.) for $110 \mathrm{~min}$ at $100 \mathrm{~V}$. Membranes were blocked for $2 \mathrm{~h}$ in blocking buffer (TBS with $0.1 \%$ Tween 20 (TBST) and 5\% BSA) at RT. The membranes were then incubated overnight at $4^{\circ} \mathrm{C}$ with primary antibody (rabbit polyclonal antineurokinin $\mathrm{B}$ receptor (NK3R, 1:500; Abbiotec)) diluted in the same buffer solution containing BSA described above. After washing the membranes extensively in washing buffer (TBS-0.1\% Tween 20), the membrane was incubated with HRP-conjugated donkey anti-rabbit IgG (1:10000, Millipore) diluted in blocking buffer for $2 \mathrm{~h}$ at $4^{\circ} \mathrm{C}$. The membrane was washed several times in the washing buffer to remove unbound secondary antibody and the signal was detected by ECL detection kit (GE Healthcare). The membranes were exposed in Image Quant LAS 4000 mini (GE Healthcare), and signals were determined by Image J software. The results were expressed according to the intensity of the signals in arbitrary densitometric units after normalization by glyceraldehyde-3phosphate dehydrogenase (GAPDH, Sigma, USA) as an internal standard.

2.6. Statistical Analysis. Values were expressed as mean \pm SEM. Statistical analysis was performed on raw data using the one-way ANOVA, with the significance concentrations of $P<0.05$ in two-tailed testing chosen. Comparisons among groups were made using the Student's $t$-test.

\section{Results}

3.1. The Day of Vaginal Opening and the Establishment of Two Regular Estrous Cycles of the Rats. The day of vaginal opening and the establishment of two regular estrous cycles were significantly advanced in $M$ than that in $N(P<0.01$, Figure 1). The day of vaginal opening and the establishment of two regular estrous cycles in CHM were significantly delayed than those of $\mathrm{M}$ and $\mathrm{MS}(P<0.01$, Figure 1$)$.

3.2. Organ Coefficients of Ovary and Uterus. On the day of prepuberty, the organ coefficients of uteri and ovaries have no significant differences among four groups. On the day of onset-puberty, the uteri and ovaries coefficients of the $M$ were increased more significantly than those of $\mathrm{N}(P<0.05, P<$ 0.05 , resp.), while they were decreased significantly in $\mathrm{CHM}$ compared with those of $\mathrm{M}(P<0.05, P<0.05$, resp. $)$ and MS; On the day of postpuberty, the ovaries coefficient of the $\mathrm{M}$ was significantly upregulated compared with that of $\mathrm{N}(P<$ 0.05); the organ coefficients of uteri and ovaries in CHM were decreased obviously compared with those of $\mathrm{M}(P<0.05$, $P<0.05$, resp.) and MS (Figure 2).

3.3. Hormone Levels in Blood of the Rats. Plasma $\mathrm{E}_{2}, \mathrm{FSH}$ levels in $\mathrm{M}$ were higher than those in $\mathrm{N}(P<0.05, P<$ 0.05 , resp.), while, the plasma $\mathrm{E}_{2}$, FSH levels in CHM were significantly decreased compared with those in $\mathrm{M}(P<0.05$, $P<0.05$, resp.) and MS. However there were no significant differences in plasma LH levels among four groups (Table 1). 


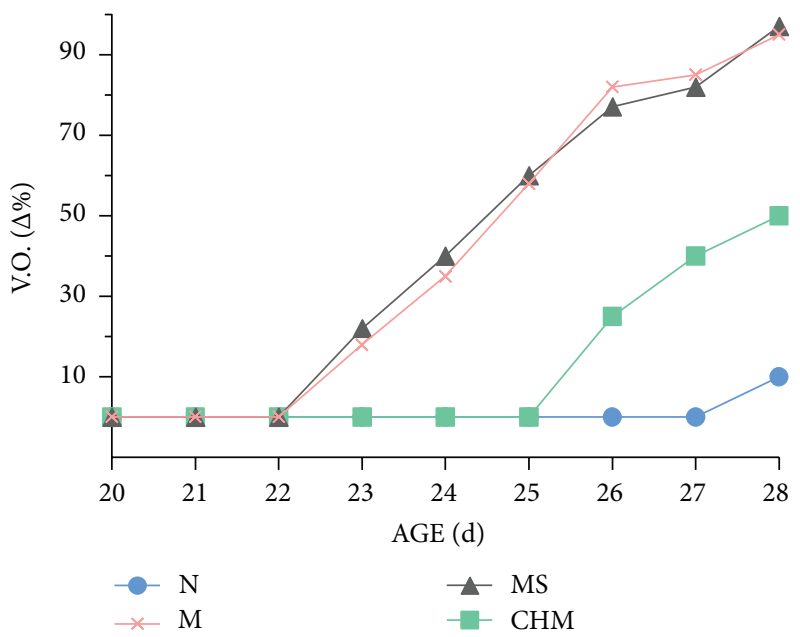

(a)

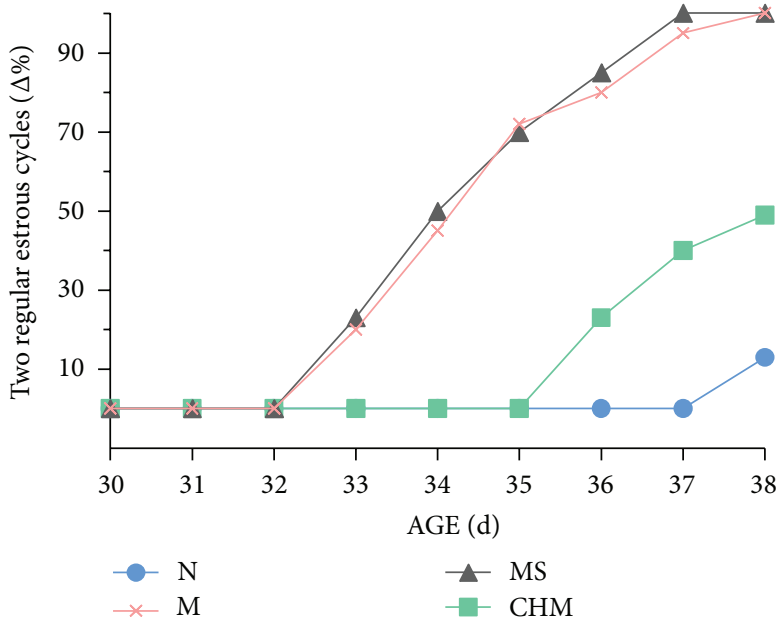

(b)

FIGURE 1: Effects of Chinese herb mixture on the day of vaginal opening and establishment of two regular estrous cyclesof female precocious rats. The day of vaginal opening and establishment of two regular estrous cycles were earlier in $\mathrm{M}$ and MS than N and then were delayed by administration of Chinese herb mixture. N: normal, M: model, MS: saline, and CHM: Chinese herb mixture.

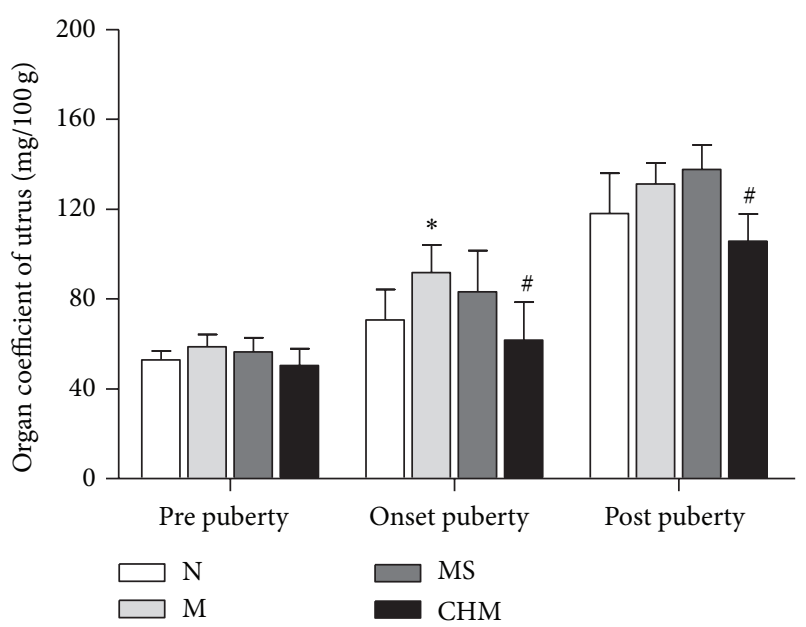

(a)

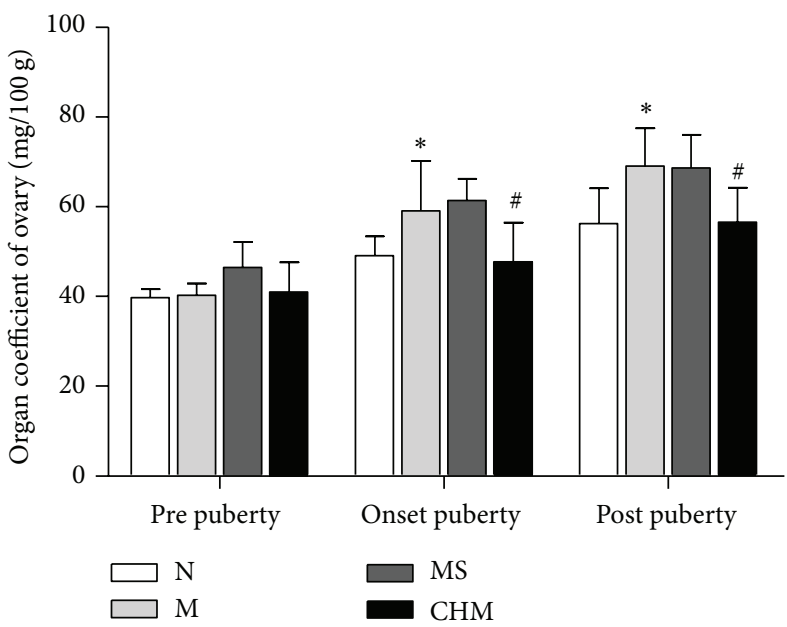

(b)

FIGURE 2: Effects of Chinese herb mixture on uterus and ovary coefficients of female precocious rats. On prepuberty, there were no significant differences among four groups; the ovary and uterus coefficients of $\mathrm{M}$ have significant predominance over $\mathrm{N}$ groups $(P<0.05)$, and CHM groups have decreased the organ coefficients in $\mathrm{M}$ of onset-puberty $(P<0.05)$ and postpuberty $(P<0.05)$. N: normal, M: model, MS: saline, and CHM: Chinese herb mixture. ${ }^{*} P<0.05$ versus $\mathrm{N} ;{ }^{\sharp} P<0.05$ versus $\mathrm{M}$.

3.4. Effects of Chinese Herbal Mixture on Hypothalamic NKB Expression by Immunofluorescence. There were few NKBir cells found in the hypothalamus in N, M, MS, and CHM on the day of prepuberty.

On the day of onset-puberty, the number of NKBir cells in ARC of $\mathrm{M}$ was obviously increased compared with those in $\mathrm{N}(P<0.01)$. The number of positive NKBir cells in ARC was significantly decreased in CHM compared with those in $\mathrm{M}(P<0.05)$. There was no statistical difference between $\mathrm{M}$ and $\mathrm{MS}$.

On the day of onset-puberty, the number of NKBir cells in MPO of M was obviously increased compared with those in $\mathrm{N}(P<0.05)$. The number of positive NKBir cells in MPO was significantly decreased in the CHM compared with those in $\mathrm{M}(P<0.05)$. There was no statistical difference between $\mathrm{M}$ and MS.

On the day of postpuberty, the NKBir cell numbers in ARC were significantly higher in $M$ than those in $\mathrm{N}(P<$ 0.05), and they decreased in CHM than those in M and MS $(P<0.05)$. In MPO, the number of NKBir cells in $\mathrm{M}$ was less than that in $\mathrm{N}(P<0.05)$, and it increased in the CHM $(P<0.05)$ than that in $\mathrm{M}$ (Figure 3$)$.

3.5. Effects of Chinese Herbal Mixture on Hypothalamic NK3R Expression by Immunofluorescence. There were few NK3Rir 


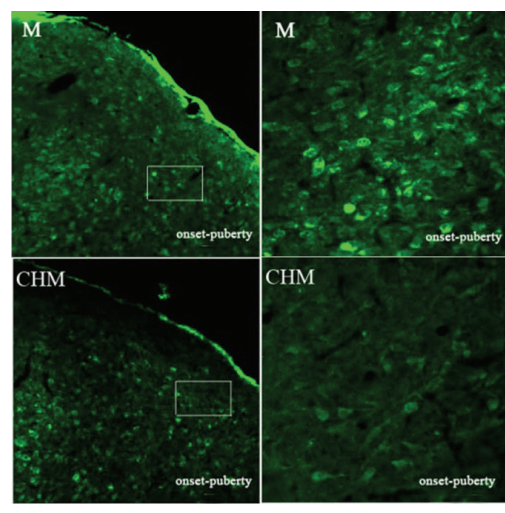

(a)
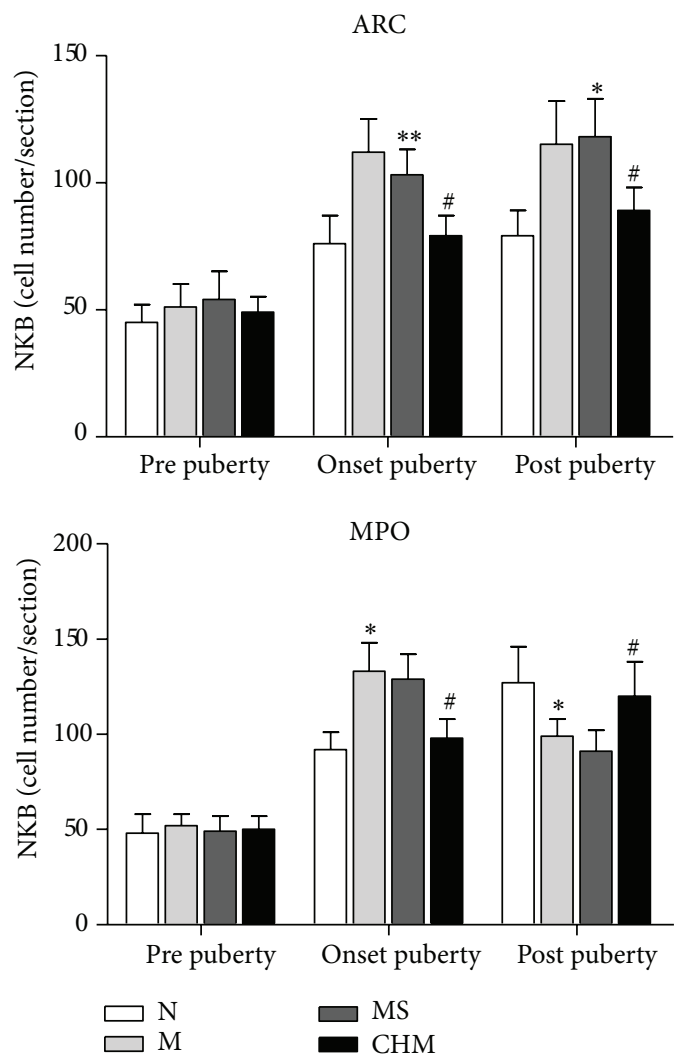

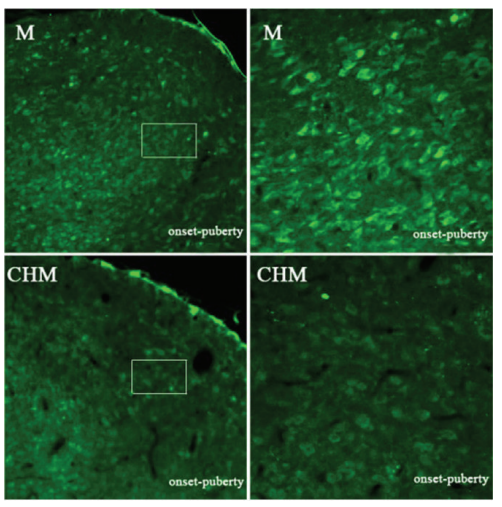

(b)
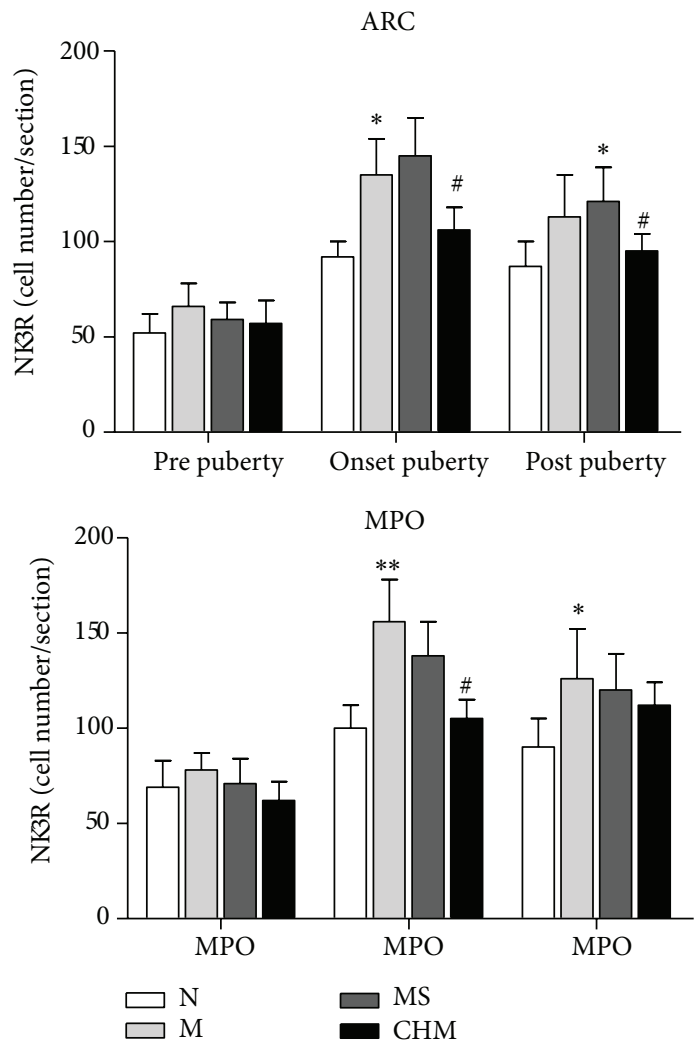

(c)

FIGURE 3: Effect of Chinese herb mixture on hypothalamic NKB/NK3R expression by immunofluorescence. (a) Representative microscopic images showing that the NKBir positive neurons located in ARC on the day of onset-puberty decreased in CHM rats compared with M ones (low magnification 10x, high magnification 40x). (b) Representative microscopic images showing that the NK3Rir positive neurons located in ARC on the day of onset-puberty decreased in CHM rats compared to M (low magnification 10x, high magnification 40x). (c) Calculated number of the NKB and NK3R neurons in the ARC and MPO in all four groups of rats ( $n=5$ per group). All observations from individual animal were averaged for that animal, and then these single numbers of each animal were used to calculate the group mean. The data is expressed as the mean with SEM. N and MS groups were not shown in the figure. N: normal, M: model, MS: saline, and CHM: Chinese herb mixture. ${ }^{*} P<0.05$ versus $\mathrm{N} ;{ }^{\#} P<0.05$ versus $\mathrm{M} ;{ }^{* *} P<0.01$ versus $\mathrm{N}$.

cells found in the hypothalamus in N, M, MS, and CHM during the prepuberty period.

On the day of onset-puberty, the number of NK3Rir cells in ARC of $M$ was obviously increased compared with that in $\mathrm{N}(P<0.05)$. The number of positive NK3Rir cells in ARC was significantly decreased in the CHM compared with that in $\mathrm{M}(P<0.05)$. There was no statistical difference between $\mathrm{M}$ and MS.

On the day of onset-puberty, the number of NK3Rir cells in MPO of M was obviously increased compared with that in $\mathrm{N}(P<0.01)$. The number of positive NK3Rir cells in MPO was significantly decreased in the CHM compared with that 
TABLE 1: Effects of Chinese herb mixture on serum $\mathrm{E}_{2}, \mathrm{FSH}$ and $\mathrm{LH}$ levels of female precocious rats.

\begin{tabular}{lccc}
\hline & $\mathrm{E}_{2}(\mathrm{pg} / \mathrm{mL})$ & $\mathrm{FSH}(\mathrm{mIU} / \mathrm{mL})$ & $\mathrm{LH}(\mathrm{mIU} / \mathrm{mL})$ \\
\hline $\mathrm{N}$ & $29.14 \pm 3.63$ & $15.58 \pm 1.11$ & $16.16 \pm 0.68$ \\
$\mathrm{M}$ & $35.93 \pm 5.45^{*}$ & $18.1 \pm 3.28^{*}$ & $17 \pm 0.98$ \\
$\mathrm{MS}$ & $35.05 \pm 2.65$ & $17.16 \pm 3.83$ & $17.34 \pm 0.79$ \\
$\mathrm{CHM}$ & $32.15 \pm 5.72^{\#}$ & $15.59 \pm 1.37^{\#}$ & $16.89 \pm 0.86$ \\
\hline
\end{tabular}

The serum level of $\mathrm{E}_{2}(P<0.05)$ and $\mathrm{FSH}(P<0.05)$ significantly increased in $\mathrm{M}$ compared with $\mathrm{N}$, and then the level of $\mathrm{E}_{2}(P<0.05)$ and FSH $(P<0.05)$ was decreased in CHM. There were no significant differences between M and MS. N: normal, M: model, MS: saline, and CHM: Chinese herb mixture.

${ }^{*} P<0.05$ versus $\mathrm{N} ;{ }^{\#} P<0.05$ versus $\mathrm{M}$.

in $\mathrm{M}(P<0.05)$. There was no statistical difference between $\mathrm{M}$ and $\mathrm{MS}$.

On the day of postpuberty, the NK3Rir cell numbers in ARC were significantly higher in $M$ than those in $\mathrm{N}(P<$ $0.05)$ and decreased in CHM than those in M and MS $(P<$ 0.05). The NK3Rir cell numbers in MPO were higher in $M$ than in $\mathrm{N}(P<0.05)$, and there was no difference between $\mathrm{CHM}$ and $\mathrm{M}$ (Figure 3 ).

3.6. Effects of Chinese Herbal Mixture on the Expression of NK3R by Western Blot. Effect of Chinese herb mixture on expression of NK3R was also detected by means of western blot. The hypothalamus NK3R expression increased significantly in $\mathrm{M}$ compared with that of $\mathrm{N}(P<0.01)$ and decreased in $\mathrm{CHM}$ compared with that in $\mathrm{M}(P<0.01)$. There were no statistical difference between M and MS (Figure 4).

3.7. Effects of Chinese Herbal Mixture on Hypothalamic NKB mRNA Expression by Real-Time PCR. Relative mRNA levels for NKB were detected by real-time reverse transcriptasePCR when puberty onset. Analysis of the NK3R mRNA concentration by the $2^{-\Delta \Delta \mathrm{Ct}}$ method and normalized to the $\beta$-actin mRNA expressed as the mean with SEM. The NKB mRNA in the $M$ increased significantly compared with that in the $\mathrm{N}(P<0.01)$, while NKB mRNA in the CHM decreased significantly compared with that in the $\mathrm{M}(P<$ 0.01) (Figure 5).

3.8. Effects of Chinese Herbal Mixture on Hypothalamic NK3R mRNA Expression by Real-Time PCR. The expression of hypothalamic NK3R mRNA at onset-puberty was detected by real-time reverse transcriptase-PCR. We used the $2^{-\Delta \Delta C t}$ method to analysis the data, and then normalized to $\beta$-actin. The ratio of NK3R to $\beta$-actin in $\mathrm{M}$ was significantly elevated compared to that in $\mathrm{N}(P<0.01)$, and it was downregulated in CHM compared to that of $\mathrm{M}(P<0.05)$. There was no statistical difference between $M$ and MS (Figure 5).

\section{Discussion}

Pubertal development occuring before the age of 8 years in girls and the age of 9 years in boys is defined as precocious puberty. Precocious puberty has a profound impact on growth, development, and psychosocial well-being of
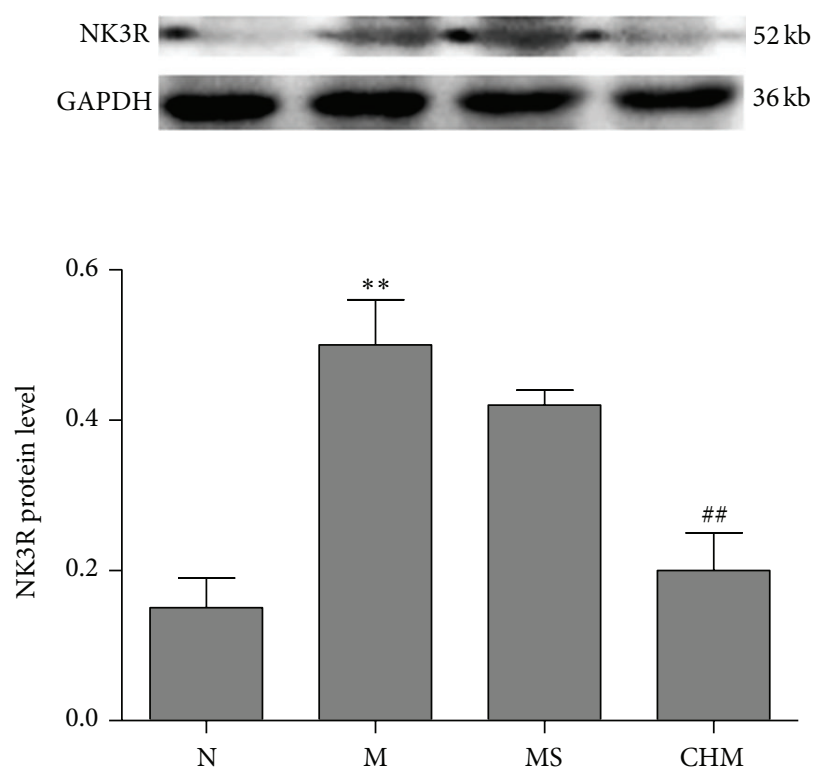

FIGURE 4: Effects of Chinese herb mixture on hypothalamus NK3R protein expression by western blot. The expression of NK3R protein was upregulated in $\mathrm{M}$ rats compared to $\mathrm{N}(P<0.01)$ and downregulated under the influence of Chinese herb mixture $(P<$ $0.01) . n=5$ per group. N: normal, M: model, MS: saline, and CHM: Chinese herb mixture. ${ }^{* *} P<0.01$ versus $\mathrm{N}$; ${ }^{\# \#} P<0.01$ versus $\mathrm{M}$.

the patients $[1,12]$. Chinese herbal mixture was proved efficient in modulating the course of puberty development [16]. But the precise mechanism of the herbal mixture is still unclear. $\mathrm{NKB} / \mathrm{NK} 3 \mathrm{R}$ signaling pathway was found to play a critical role in puberty development. The present study is to investigate the influence of nourishing "Yin"-removing "Fire" Chinese herbal mixture on NKB/NK3R expression in precocious puberty.

Our experiment showed that the day of vaginal opening and the establishment of two regular cycles were obviously delayed, after administration of CPP model rats with nourishing "Yin"-removing "Fire" Chinese herbal mixture. On the day of onset-puberty, the serum levels of E2 and FSH in CHM were also significantly reduced by Chinese herbal mixture. The Chinese herbal mixture could postpone the development of uterine and ovarian in CPP rats. To further explore the role of $\mathrm{NKB}$ and $\mathrm{NK} 3 \mathrm{R}$ in precocious puberty, the expression of $\mathrm{NKB}$ and $\mathrm{NK} 3 \mathrm{R}$ in the arcuate nucleus (ARC) and medial preoptic (MPO) was observed at different stages of puberty. We found that the number of NKB/NK3R positive cells in hypothalamus of $\mathrm{M}$ was significantly increased upon puberty onset; the expression of NKB/NK3R mRNA and $\mathrm{NK} 3 \mathrm{R}$ protein was also upregulated on the day of onsetpuberty, indicating that the $\mathrm{NKB} / \mathrm{NK} 3 \mathrm{R}$ signaling might be involved in the activation of puberty and advance of puberty. The increased NKB cell number might play an exciting role on $\mathrm{GnRH}$ secretion by projecting to the GnRH neurons at $\mathrm{ME}$ [3] upon puberty onset. On the day of establishment of two regular estrous cycles, the expression trends of NKB/NK3R in ARC were consistent with the day of onset-puberty; probably for that the ARC maintained a basic secretion of 


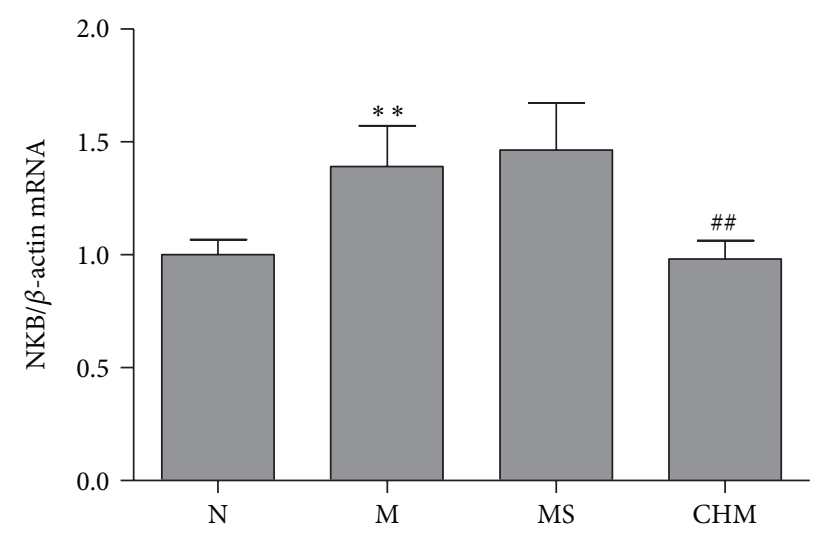

(a)

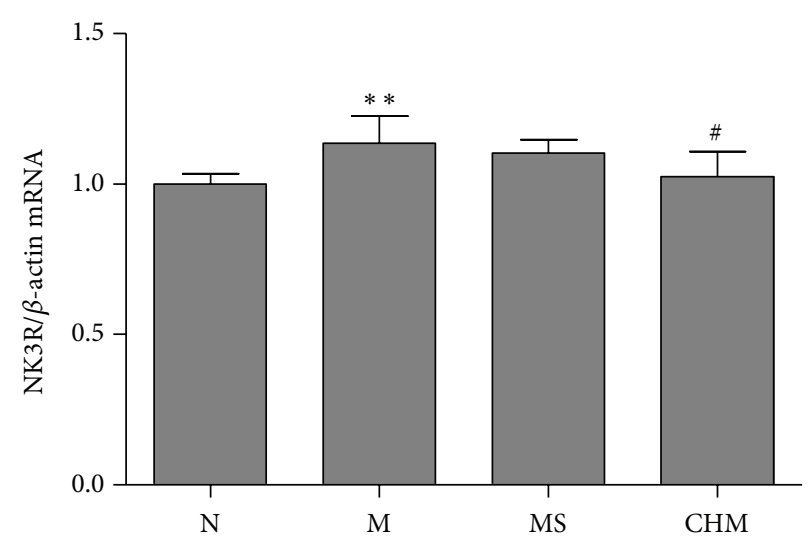

(b)

FIGURE 5: Effects of Chinese herb mixture on hypothalamic NKB/NK3R mRNA expression by real-time PCR. (a) The expression of NKB mRNA in $\mathrm{M}$ has significantly increased compared to $\mathrm{N}(P<0.01)$ and decreased in CHM compared to $\mathrm{M}(P<0.01)$. (b) The picture shows that the expression of NK3R mRNA in M was higher than in $\mathrm{N}(P<0.01)$, and that Chinese herb mixture was downregulated NK3R mRNA expression compared to $\mathrm{M}(P<0.05)$. Results analysis used the ratio of NKB or NK3R/ $\beta$-actin $(n=5$ per group) expressed as the mean with SEM. N: normal, M: model, MS: saline, and CHM: Chinese herb mixture. ${ }^{* *} P<0.01$ versus $\mathrm{N}$; ${ }^{\#} P<0.05$ versus $\mathrm{M}$; ${ }^{\# \#} P<0.01$ versus $\mathrm{M}$.

GnRH, which did not change significantly during the estrus cycle. In MPO, the number of NKBir cells was significantly decreased in $\mathrm{M}$ compared to that of $\mathrm{N}$ on postpuberty, maybe because the MPO area mainly maintained the cyclical secretion of $\mathrm{GnRH}$, and was strongly influenced by serum estrogen level [20,21]. ARC and MPO areas were important in the modulation of hypothalamus neuroendocrine function and were closely related to the regulation of $\mathrm{GnRH}$ neurons $[7,22]$. The different expressions of NKB/NK3R in ARC and MPO indicated that hypothalamic ARC and MPO played different roles in promoting puberty initiate and normal sexual cycles and finally maintained the normal development of puberty together.

After giving precocious puberty rats with nourishing "Yin"-removing "Fire" Chinese herbal mixture, the number of NKB/NK3Rir neurons in ARC and MPO was decreased significantly on the day of onset-puberty; the NKB/NK3R mRNA and NK3R protein expression in hypothalamus was also reduced significantly, which showed that the nourishing "Yin"-removing "Fire" Chinese herbal mixture not only reduced the hypothalamus TAC3 and TAC3R transcription during precocious rats, downregulated $\mathrm{NKB}$ and NK3R mRNA synthesis, but also inhibited the release of NKB and expression of NK3R. NKB neurons have direct connection with GnRH neurons and fibers [3], and there are NK3R expression on GnRH neurons [6], we speculated that the nourishing "Yin"-removing "Fire" Chinese herbal mixture may influence the GnRH release through inhibiting NKB and NK3R expression and then delay the HPGA initiation. The role of NKB involved in the therapeutic effects of Chinese herbal mixture on precocious puberty rats is implemented by its receptor NK3R.

\section{Conclusion}

$\mathrm{NKB} / \mathrm{NK} 3 \mathrm{R}$ signaling might be involved in the advance onset-puberty in precocious puberty and the regulation of nourishing "Yin"-removing "Fire" Chinese herb mixture on the abnormal function of HPGA in precocious puberty rats. The potential mechanism of the herb mixture needs to be further revealed so as to bring evidence to clinical exercise.

\section{Conflict of Interests}

The authors declare that there is no conflict of interests regarding the publication of this paper.

\section{Authors' Contribution}

Shiran Wang and Zhanzhuang Tian designed the study. Shiran Wang and Liting Zhu performed the animal and molecular experiments. Shiran Wang drafted the paper. Zhanzhuang Tian gave good suggestion and advice for paper writing and revising. Jian Yu participated in the coordination work of the study. All authors read and approved the final paper.

\section{Acknowledgment}

This project was financially supported by the National Natural Science Foundation (no. 30973814 and no. 81273894).

\section{References}

[1] G. Prété, A.-C. Couto-Silva, C. Trivin, and R. Brauner, "Idiopathic central precocious puberty in girls: presentation factors," BMC Pediatrics, vol. 8, article 27, 2008.

[2] A. K. Topaloglu, "Neurokinin B signaling in puberty: human and animal studies," Molecular and Cellular Endocrinology, vol. 324, no. 1-2, pp. 64-69, 2010.

[3] S. J. Krajewski, M. J. Anderson, L. Iles-Shih, K. J. Chen, H. F. Urbanski, and N. E. Rance, "Morphologic evidence that neurokinin B modulates gonadotropin-releasing hormone secretion via neurokinin 3 receptors in the rat median eminence," 
Journal of Comparative Neurology, vol. 489, no. 3, pp. 372-386, 2005.

[4] J. Young, J. Bouligand, B. Francou et al., "TAC3 and TACR3 defects cause hypothalamic congenital hypogonadotropic hypogonadism in humans," Journal of Clinical Endocrinology and Metabolism, vol. 95, no. 5, pp. 2287-2295, 2010.

[5] L. G. Silveira, C. Tusset, and A. C. Latronico, "Impact of mutations in kisspeptin and neurokinin B signaling pathways on human reproduction," Brain Research, vol. 1364, pp. 72-80, 2010.

[6] K.-I. Noritake, T. Matsuoka, T. Ohsawa et al., "Involvement of neurokinin receptors in the control of pulsatile luteinizing hormone secretion in rats," Journal of Reproduction and Development, vol. 57, no. 3, pp. 409-415, 2011.

[7] V. M. Navarro, J. M. Castellano, S. M. McConkey et al., "Interactions between kisspeptin and neurokinin B in the control of $\mathrm{GnRH}$ secretion in the female rat," The American Journal of Physiology -Endocrinology and Metabolism, vol. 300, no. 1, pp. E202-E210, 2011.

[8] K. Sakamoto, K. Murata, Y. Wakabayashi et al., "Central administration of neurokinin $\mathrm{B}$ activates kisspeptin/NKB neurons in the arcuate nucleus and stimulates luteinizing hormone secretion in ewes during the non-breeding season," Journal of Reproduction and Development, vol. 58, no. 6, pp. 700-706, 2012.

[9] V. M. Navarro, "New insights into the control of pulsatile GnRH release: the role of Kiss1/neurokinin B neurons," Frontiers in Endocrinology, vol. 3, article 48, 2012.

[10] S. Ramaswamy, S. B. Seminara, B. Ali, P. Ciofi, N. A. Amin, and T. M. Plant, "Neurokinin B stimulates GnRH release in the male monkey (Macaca mulatta) and is colocalized with kisspeptin in the arcuate nucleus," Endocrinology, vol. 151, no. 9, pp. 4494$4503,2010$.

[11] Y. Wakabayashi, T. Nakada, K. Murata et al., "Neurokinin B and dynorphin A in kisspeptin neurons of the arcuate nucleus participate in generation of periodic oscillation of neural activity driving pulsatile gonadotropin-releasing hormone secretion in the goat," Journal of Neuroscience, vol. 30, no. 8, pp. 3124-3132, 2010.

[12] L. Lazar, R. Kauli, A. Pertzelan, and M. Phillip, "Gonadotropinsuppressive therapy in girls with early and fast puberty affects the pace of puberty but not total pubertal growth or final height," Journal of Clinical Endocrinology and Metabolism, vol. 87, no. 5, pp. 2090-2094, 2002.

[13] V. V. Khadilkar, A. V. Khadilkar, and G. B. Maskati, "Growth hormone and GnRHa combination therapy in the management of precocious puberty," Indian Pediatrics, vol. 42, no. 1, pp. 5760, 2005.

[14] C. A. Longui, C. Kochi, L. E. P. Calliari et al., "Near-final height in patients with congenital adrenal hyperplasia treated with combined therapy using gh and GnRHa," Arquivos Brasileiros de Endocrinologia e Metabologia, vol. 55, no. 8, pp. 661-664, 2011.

[15] J. S. Fuqua, "Treatment and outcomes of precocious puberty: an update," Journal of Clinical Endocrinology and Metabolism, vol. 98, no. 6, pp. 2198-2207, 2013.

[16] Z. Tian, H. Zhao, Y. Sun, D. Cai, and B. Chen, "Evaluation of the true precocious puberty rats induced by neonatal administration of Danazol: therapeutic effects of nourishing "Yin"removing "Fire" Chinese Herb Mixture," Reproductive Biology and Endocrinology, vol. 3, article 38, 2005.

[17] D. P. Cai, Z. Y. Ji, and Y. M. Shi, "Clinical study on treatment of female idiopathic precocious puberty with combined therapy of Chinese medicine and megestrol acetate," Chinese Journal of Integrated Traditional and Western Medicine, vol. 21, no. 10, pp. 732-735, 2001.

[18] Y. Sun, G. N. Perry, J. Yu, B. Chen, and Z. Tian, "Effect of nourishing "Yin"-removing "Fire" Chinese herbal mixture on hypothalamic kisspeptin expression in female precocious rats," Journal of Ethnopharmacology, vol. 127, no. 2, pp. 274-279, 2010.

[19] H. Morishita, M. Takemoto, H. Kondo, K. Higuchi, and T. Aono, "Induction of true precocious puberty by neonatal treatment with danazol in female rats," Neuroscience Letters, vol. 157, no. 1, pp. 33-36, 1993.

[20] N. E. Rance, "Menopause and the human hypothalamus: evidence for the role of kisspeptin/neurokinin B neurons in the regulation of estrogen negative feedback," Peptides, vol. 30, no. 1, pp. 111-122, 2009.

[21] T. L. Dellovade and I. Merchenthaler, "Estrogen regulation of neurokinin B gene expression in the mouse arcuate nucleus is mediated by estrogen receptor $\alpha$," Endocrinology, vol. 145, no. 2, pp. 736-742, 2004.

[22] S. J. Krajewski, M. C. Burke, M. J. Anderson, N. T. Mcmullen, and N. E. Rance, "Forebrain projections of arcuate neurokinin b neurons demonstrated by anterograde tract-tracing and monosodium glutamate lesions in the rat," Neuroscience, vol. 166, no. 2, pp. 680-697, 2010. 


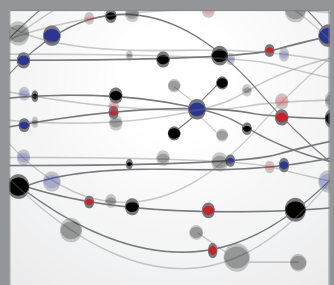

The Scientific World Journal
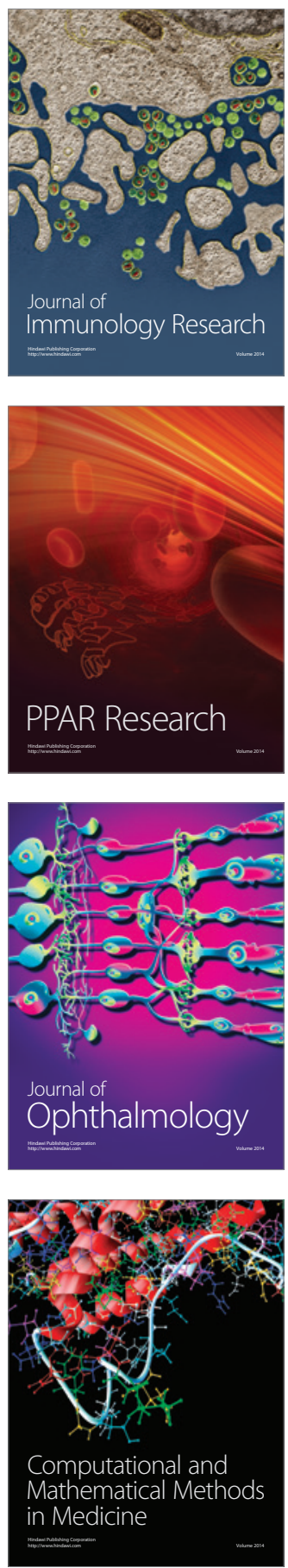

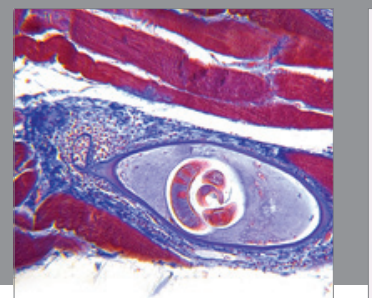

Gastroenterology

Research and Practice
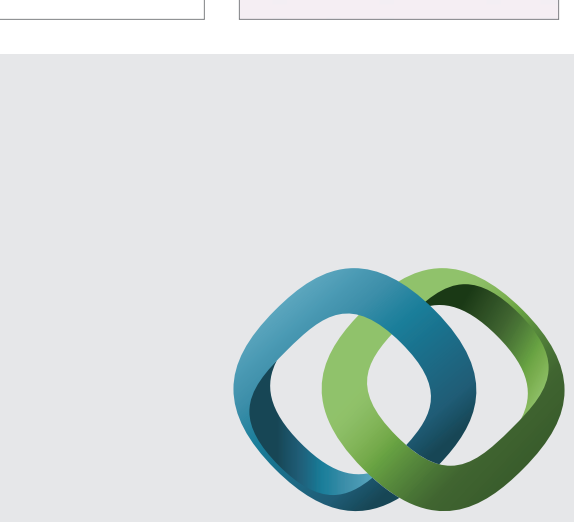

\section{Hindawi}

Submit your manuscripts at

http://www.hindawi.com
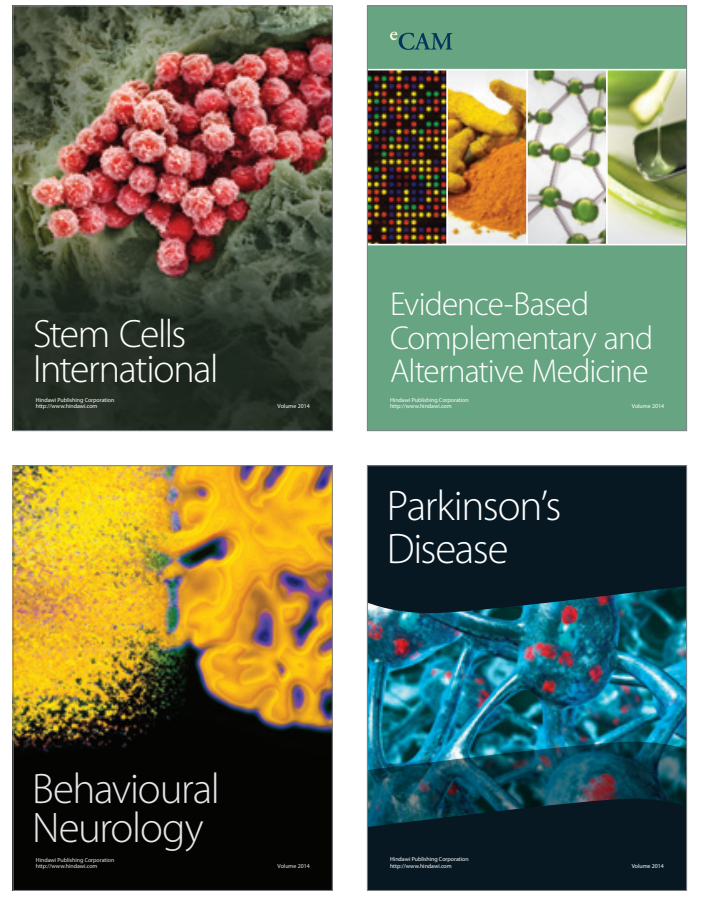
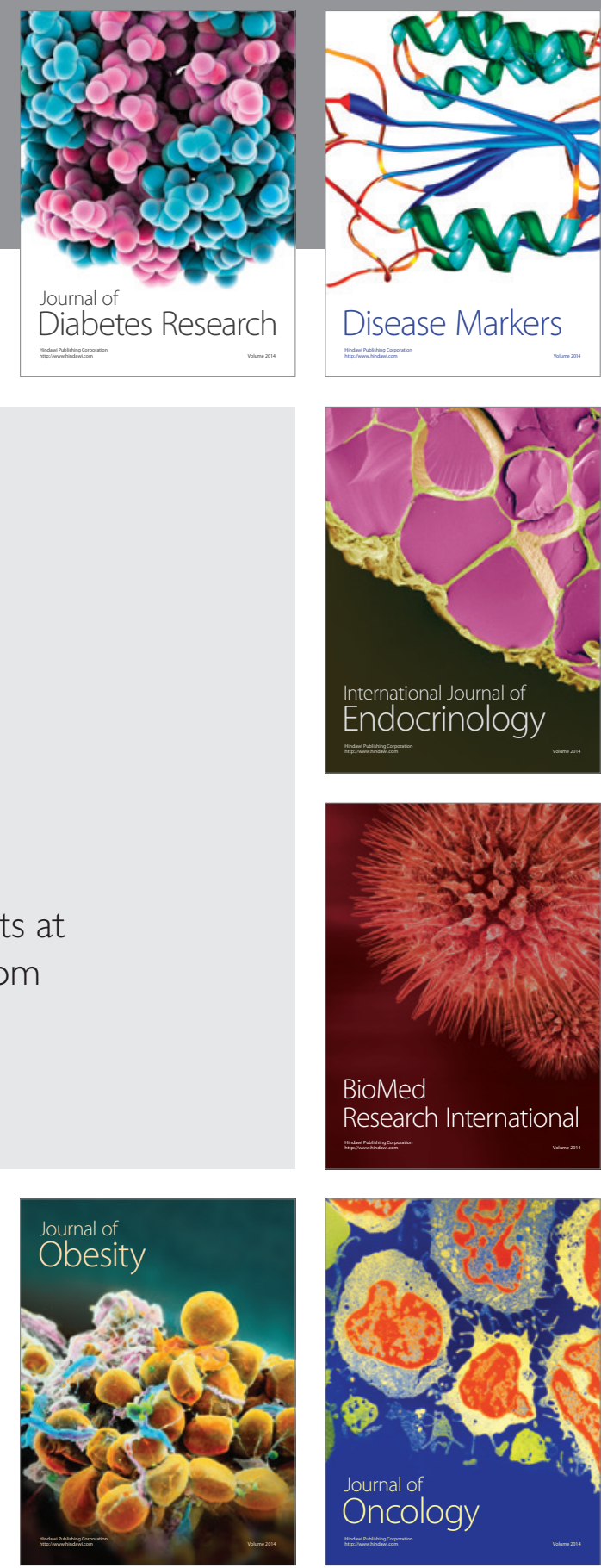

Disease Markers
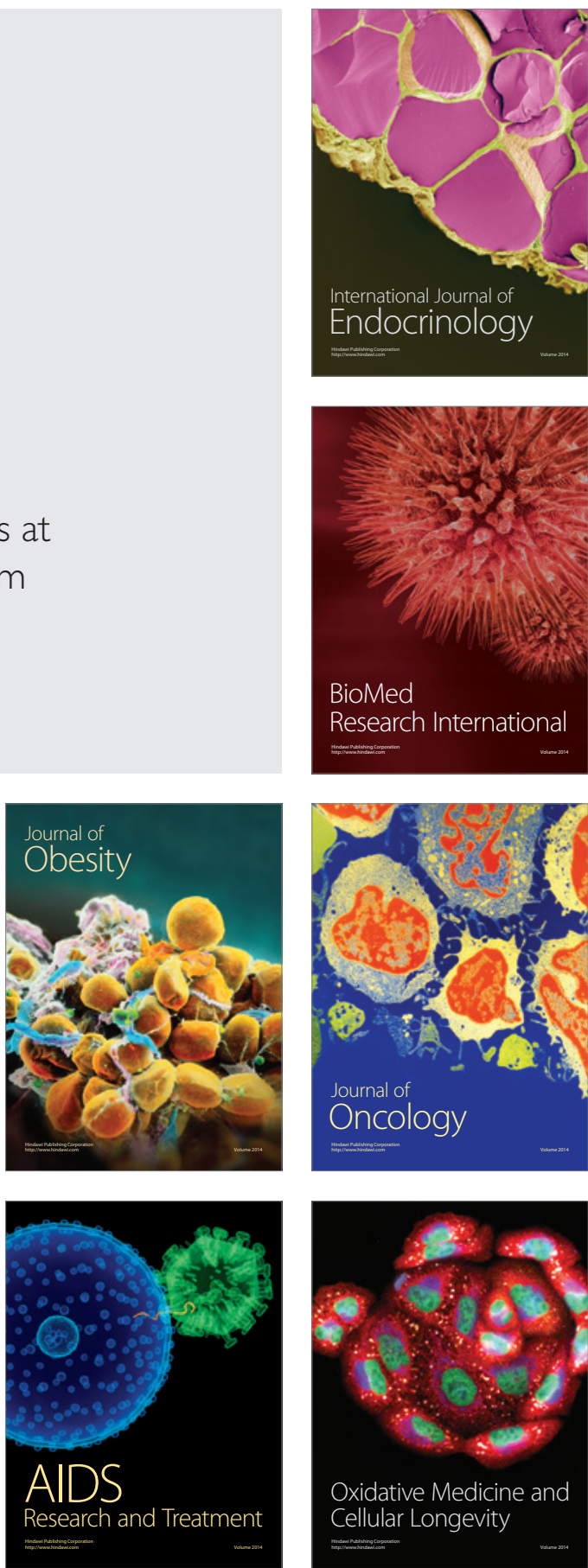